\title{
The persistence of prey-predator model with competition hosts
}

\author{
Azhar Abbas Majeed ${ }^{1}$, Rami Raad Saadi ${ }^{2}$ \\ ${ }^{1}$ Baghdad University, Iraq \\ ${ }^{2}$ Iraqi University, Iraq \\ *Corresponding author E-mail: azhar_abbas_m@yahoo.com
}

Copyright $\odot 2015$ Azhar Abbas Majeed, Rami Raad Saadi. This is an open access article distributed under the Creative Commons Attribution License, which permits unrestricted use, distribution, and reproduction in any medium, provided the original work is properly cited.

\begin{abstract}
In this paper, the conditions of the occurrence of persistence of a mathematical model consists from four-species SynEcosymbiosis involves a competition hosts for prey are established. Finally, in order to confirm our obtained analytical results, numerical simulations have been done for a hypothetical set of parameter values.
\end{abstract}

Keywords: Equilibrium Point; Persistence; Lyapunov Average Function.

\section{Introduction}

The dynamic relationship between predators and their prey has long been and will continue to be one of the dominant themes in both ecology and mathematical ecology due to the universal existence and importance. An important and ubiquitous problem in predator-prey theory and related topics in mathematical ecology, concerns the long term coexistence (or persistence) of species, for example, see [1-8].

Freedman and Waltman [9] considered three-level food webs-two competing predators feeding on a single prey and a single predator feeding on two competing prey species. They obtained criteria for the system to be persist.

Rami \& Raid [10] proposed and analyzed a prey-predator model with four Syniecological system with Holling type-II functional response, they obtained a set of sufficient and necessary condition, which guarantee the lacal and global stability of this system.

In this paper, however, we will establish the conditions of the occurrence of persistence of a mathematical model proposed by Rami \& Raid [10].

\section{Mathematical model[10]}

An ecological model of four species Syn-Ecosymbiosis, comprising of prey-predator, commensalisms and competition, model is proposed in [10].

$$
\begin{aligned}
& \frac{d N_{1}}{d T}=r_{1} N_{1}\left(1-\frac{N_{1}}{k_{1}}\right)-\frac{a_{1} N_{1}}{b+N_{1}} N_{2}+c N_{1} N_{3} \\
& \frac{d N_{2}}{d T}=e \frac{a_{1} N_{1}}{b+N_{1}} N_{2}-d_{1} N_{2}-d_{2} N_{2}^{2} \\
& \frac{d N_{3}}{d T}=r_{2} N_{3}\left(1-\frac{N_{3}}{k_{2}}\right)-\alpha_{1} N_{3} N_{4} \\
& \frac{d N_{4}}{d T}=r_{3} N_{4}\left(1-\frac{N_{4}}{k_{3}}\right)-\alpha_{2} N_{3} N_{4}
\end{aligned}
$$

Where $0<e<1$ represents the conversion rate. 
This model consists of a prey (for example, Anemone) whose population density at time $T$ denoted by $N_{1}$, the predator (for example, Butterfly fish) whose population density at time $T$ denoted by $N_{2}$, the host (for example, Hermit crabs) whose population density at time $T$ denoted by $N_{3}$, and the host's competitor species (for example, other type of Hermit crabs) whose population density at time $T$ denoted by . All the parameters ar Moreover assumed to be positive and described as given in [10].

Now, for further simplification of the system (2), the following dimensionless variables are used in [10].

$$
\begin{aligned}
& t=r_{1} T, x=\frac{N_{1}}{k_{1}}, y=\frac{N_{2}}{k_{1}}, z=\frac{c N_{3}}{r_{1}}, w=\frac{\alpha_{1} N_{4}}{r_{1}}, u_{1}=\frac{a_{1}}{r_{1}} \\
& u_{2}=\frac{b}{k_{1}}, u_{3}=\frac{d_{1}}{r_{1}}, u_{4}=\frac{d_{2} k_{1}}{r_{1}}, u_{5}=\frac{r_{2}}{r_{1}}, \\
& u_{6}=\frac{r_{1}}{c k_{2}}, u_{7}=\frac{r_{3}}{r_{1}}, u_{8}=\frac{r_{1}}{\alpha_{1} k_{3}}, u_{9}=\frac{\alpha_{2}}{c}
\end{aligned}
$$

Thus, system (2) can be turned into the following dimensionless form:

$$
\begin{aligned}
& \frac{d x}{d t}=x\left[(1-x)-\frac{u_{1} y}{u_{2}+x}+z\right]=x f_{1}(x, y, z, w) \\
& \frac{d y}{d t}=y\left[\frac{e u_{1} x}{u_{2}+x}-u_{3}-u_{4} y\right]=y f_{2}(x, y, z, w) \\
& \frac{d z}{d t}=z\left[u_{5}\left(1-u_{6} z\right)-w\right]=z f_{3}(x, y, z, w) \\
& \frac{d w}{d t}=w\left[u_{7}\left(1-u_{8} w\right)-u_{9} z\right]=w f_{4}(x, y, z, w)
\end{aligned}
$$

With $x(0) \geq 0, y(0) \geq 0, z(0) \geq 0$ and It is observed that $\mathrm{t}$, . e number of parameters have been reduced from fourteen in the system (1) to ten in the system (2). Obviously the interactObviously,ions of the system (2) are continuous and have continuous partial de been continuous the following positive four dimensional space:

Therefore these functions are Lipschitzian on $R_{+}^{4}$, and hence the solution of the system (2) exists and is unique. Further, in the following theorem, the boundedness of the solution of the system (2) in $R_{+}^{4}$ is established by [10].

Theorem 1: All the solutions of system (2) which initiate in $R_{+}^{4}$ are uniformly bounded

\section{The stability analysis of equilibrium points of system (2) [10]}

The four-species Syn-Ecosymiois model given by the system (2) has at most twelve equilibrium points, which are mentioned with their existence conditions in [10] as in the following:

1) The equilibrium points $E_{0}=(0,0,0,0), E_{1}=(1,0,0,0), E_{2}=\left(0,0, \frac{1}{u_{6}}, 0\right)$,

$E_{3}=\left(0,0,0, \frac{1}{u_{8}}\right), E_{4}=(\widehat{x}, \widehat{y}, 0,0)$ and $E_{5}=(0,0, \tilde{z}, \tilde{w})$, where $\tilde{w}=\frac{u_{5}\left(u_{6} u_{7}-u_{9}\right)}{u_{5} u_{6} u_{7} u_{8}-u_{9}}$ and $\tilde{z}=\frac{u_{7}\left(u_{5} u_{8}-1\right)}{u_{5} u_{6} u_{7} u_{8}-u_{9}}$ are unstable points for more details see [10].

2) The third planar equilibrium point $E_{6}=(\bar{x}, 0, \bar{z}, 0)=\left(\frac{u_{6}+1}{u_{6}}, 0, \frac{1}{u_{6}}, 0\right)$ always exists in Int.R $R_{+}^{2}$ of $x z-$ plane, $E_{6}$ is locally asymptotically stable. If the following conditions hold

$e u_{1}\left(1+u_{6}\right)<u_{3}\left(u_{2} u_{6}+u_{6}+1\right)$

$u_{6} u_{7}<u_{9}$

However, it is a saddle point otherwise.

6-The fourth planar equilibrium point $E_{7}=(\overline{\bar{x}}, 0,0, \overline{\bar{w}})=\left(1,0,0, \frac{1}{u_{8}}\right)$ always exists in Int. $R_{+}^{2}$ of $x w-$ plane, $E_{7}$ is locally asymptotically stable. If the following conditions hold

$e u_{1}<u_{3}\left(u_{2}+1\right)$ 
$u_{5} u_{8}<1$

However, it is a saddle point otherwise.

3) The first three species equilibrium points where.

$\breve{y}=\frac{u_{6}\left[\left(u_{2}+\breve{x}\right)(1-\breve{x})\right]+\left(u_{2}+\breve{x}\right)}{u_{1} u_{6}}$ And $\breve{z}=\frac{1}{u_{6}}$

And $\widetilde{x}$ is positive constant, is locally asymptotically stable in the $R_{+}^{4}$ if the following conditions are satisfied

$\frac{u_{1} \breve{y}}{\left(u_{2}+\widetilde{x}\right)^{2}}<1$

$u_{6} u_{7}<u_{9}$

However, it is a saddle point otherwise.

4) The second three species equilibrium point $E_{9}=(\hat{x}, \hat{y}, 0, \hat{w})$

Where $\hat{y}=\frac{(1-\hat{x})\left(u_{2}+\hat{x}\right)}{u_{1}}, \hat{w}=\frac{1}{u_{8}}$, and $0<\hat{x}<1$

Is locally asymptotically stable in the $R_{+}^{4}$ if the following conditions are satisfied:

$\frac{u_{1} \hat{y}}{\left(u_{2}+\hat{x}\right)^{2}}<1$

$u_{5} u_{8}<1$

However, it is a saddle point otherwise.

5) The third three species equilibrium point $E_{10}=\left(x^{\bullet}, 0, z^{\bullet}, w^{\bullet}\right)$

Where $x^{\bullet}=\frac{u_{5} u_{6} u_{7} u_{8}-u_{9}+u_{7}\left(u_{5} u_{8}-1\right)}{u_{5} u_{6} u_{7} u_{8}-u_{9}}, z^{\bullet}=\tilde{z}, w^{\bullet}=\tilde{w}$

$E_{10}$ Is locally asymptotically stable in the $R_{+}^{4}$ if the following conditions are satisfied

$u_{5} u_{6} u_{7} u_{8}>u_{9}$

$e u_{1} x^{\bullet}<u_{3}\left(u_{2}+x^{\bullet}\right)$

However, it is a saddle point otherwise.

6) Finally the positive (coexistence) equilibrium point $E_{11}=\left(x^{*}, y^{*}, z^{*}, w^{*}\right)$

Where $z^{*}=\tilde{z}, w^{*}=\tilde{w}$

$y=\frac{\left(u_{2}+x\right)\left[s_{2}(1-x)+u_{7} s_{1}\right]}{u_{1} s_{2}}$

$E_{11}$ Is locally asymptotically stable in the if the following conditions are satisfied.

$\frac{u_{1} y^{*}}{\left(u_{2}+x^{*}\right)^{2}}<1$

$u_{5} u_{6} u_{7} u_{8}>u_{9}$

However, it is a saddle point otherwise. 


\section{The persistence of system (2)}

In general, persistence is a global property of a dynamical system; it is not dependence upon interior solution space structure but is dependent upon solution behavior near extinction boundaries (boundary planes).From From the biological point of view, persistence of a system means the survival of all population of the system in future time. However, mathematically it means that strictly positive solutions do not have omega limit set on the boundary of the non-negative cone [11].Accordingly, if the dynamic system does not persist, then the solution have omega limit set on the boundary of the nonnegative cone, and hence the dynamic system faces extinction. Now, before examine the persistence of stage structure model given by system (2) by using the method of average Lyapunov function as given in [12], we need to study the global dynamics in the boundary planes $x z, x w$, and in the Int. $R_{+}^{3}$ as shown in the following theorems.

Theorem 2: Suppose that the equilibrium point $E_{6}=(\bar{x}, 0, \bar{z}, 0)=\left(\frac{u_{6}+1}{u_{6}}, 0, \frac{1}{u_{6}}, 0\right)$ is locally asymptotically stable in the Int $R_{+}^{2}$ then it is a globally asymptotically stable in the Int $R_{+}^{2}$ of the $x z$-plane.

Proof: We will proof the theorem in the. For any initial value Clearly in the Int. $R_{+}^{2}$ of $x z$-plane, system (2) reduces to the following subsystem

$$
\begin{aligned}
& \frac{d x}{d t}=x[(1-x)+z]=h_{1}(x, z) \\
& \frac{d z}{d t}=z\left[u_{5}\left(1-u_{6} z\right)\right]=h_{2}(x, z)
\end{aligned}
$$

Obviously $E_{6}$ represents the positive equilibrium point of subsystem (2.3) in the Int $R_{+}^{2}$ Of $x z$-plane. Assume that $H(x, z)=\frac{1}{x z}$. Clearly, $H(x, z)$ is a $C^{1}$ function and is a positive for all. Further

$$
\begin{aligned}
\Delta(x, z) & =\frac{\partial}{\partial x}\left(H h_{1}\right)+\frac{\partial}{\partial z}\left(H h_{2}\right) \\
& =-\frac{1}{z}-\frac{u_{5} u_{6}}{x} .
\end{aligned}
$$

Note that $\Delta(x, z)$ does not change sign and is not identically zero in the Int $R_{+}^{2}$ of the $x z$-plane. Then according to Bendixson -Dualic criterion subsystem (2.3) has no periodic dynamic in the interior of positive quadrant of $x z$-plane. Further, since $E_{6}$ is the only positive equilibrium point of subsystem (2.3) in the interior of positive quadrant of $x z$ plane. Hence according to Poincare-Bendixson theorem $E_{6}$ is a globally asymptotically stable in the interior of positive quadrant.

Theorem 3: Suppose that the equilibrium point $E_{7}=\left(1,0,0, \frac{1}{u_{8}}\right)$ is locally asymptotically stable in the Int $R_{+}^{2}$ then it is a globally asymptotically stable in the Int. $R_{+}^{2}$ of the $x w$-plane.

Proof: We will proof the theorem in the. For any initial value clearly in the $I n t \cdot R_{+}^{2}$ of $x w$-plane, system (2) reduces to the following subsystem

$$
\begin{aligned}
& \frac{d x}{d t}=x(1-x)=h_{1}(x, w) \\
& \frac{d w}{d t}=w\left[u_{7}\left(1-u_{8} w\right)\right]=h_{2}(x, w)
\end{aligned}
$$

Obviously $E_{7}$ represents the positive equilibrium point of subsystem (2.4) in the Int $R_{+}^{2}$ of $x w$-plane. Assume that $H(x, w)=\frac{1}{x w}$. Clearly, $H(x, w)$ is a $C^{1}$ function and is a positive for all. Further 


$$
\begin{aligned}
\Delta(x, w) & =\frac{\partial}{\partial x}\left(H h_{1}\right)+\frac{\partial}{\partial w}\left(H h_{2}\right) \\
& =-\frac{1}{w}-\frac{u_{7} u 8}{x}
\end{aligned}
$$

Note that $\Delta(x, w)$ does not change sign and is not identically zero in the Int. $R_{+}^{2}$ of the $x w$-plane. Then according to Bendixson -Dualic criterion subsystem (2.4) has no periodic dynamic in the interior of positive quadrant of $x w$-plane. Further, since $E_{7}$ is the only positive equilibrium point of subsystem (2.4) in the interior of positive quadrant of $x w-$ plane. Hence according to Poincare-Bendixson theorem $E_{7}$ is a globally asymptotically stable in the interior of positive quadrant.

Theorem 4: Assume that, the equilibrium point $E_{8}=(\breve{x}, \breve{y}, \breve{z}, 0)=\left(\breve{x}, \breve{y}, \frac{1}{u_{6}}, 0\right)$ of the system (2) is locally asymptotically stable in the Int. $R_{+}^{3}$ and the following conditions hold.

$$
\begin{aligned}
& \frac{u_{1} \breve{y}}{u_{2}\left(u_{2}+\breve{x}\right)}<1 \\
& \left(\frac{e u_{1} u_{2}-u_{1} u_{2}-u_{1} \breve{x}}{\left(u_{2}-x\right)\left(u_{2}+\breve{x}\right)}\right)^{2}<2 u_{4}\left(1-\frac{u_{1} \breve{y}}{u_{2}\left(u_{2}+\breve{x}\right)}\right) \\
& 1<2 u_{5} u_{6}\left(1-\frac{u_{1} \breve{y}}{u_{2}\left(u_{2}+\widetilde{x}\right)}\right)
\end{aligned}
$$

Then the equilibrium point $E_{8}$ of the system (2) is globally asymptotically stable in the $I n t . R_{+}^{3}$ of the xyz-space.

Proof: We will proof the theorem in the. For any initial value clearly in the Int.$R_{+}^{3}$ of xyz-space, system (2) reduces to the following subsystem.

$$
\begin{aligned}
& \frac{d x}{d t}=x\left[(1-x)-\frac{u_{1} y}{u_{2}+x}+z\right]=x f_{1}(x, y, z) \\
& \frac{d y}{d t}=y\left[\frac{e u_{1} x}{u_{2}+x}-u_{3}-u_{4} y\right]=y f_{2}(x, y, z) \\
& \frac{d z}{d t}=z\left[u_{5}\left(1-u_{6} z\right)\right]=z f_{3}(x, y, z)
\end{aligned}
$$

Now, consider the following function

$$
V_{1}(x, y, z)=\left(x-\breve{x}-\breve{x} \ln \frac{x}{\breve{x}}\right)+\left(y-\breve{y}-\breve{y} \ln \frac{y}{\breve{y}}\right)+\left(z-\breve{z}-\bar{z} \ln \frac{z}{\breve{z}}\right)
$$

Clearly, $V_{1}: R_{+}^{3} \rightarrow R$ is a $C^{1}$ positive definite function? Now by differentiating $V_{1}$ with respect to time $\mathrm{t}$ and doing some algebraic manipulation, gives that:

$$
\begin{gathered}
\frac{d V_{1}}{d t} \leq-\left(1-\frac{u_{1} \breve{y}}{u_{2}\left(u_{2}+\breve{x}\right)}\right)(x-\breve{x})^{2}+\left(\frac{e u_{1} u_{2}-u_{1} u_{2}-u_{1} \breve{x}}{\left(u_{2}-x\right)\left(u_{2}+\breve{x}\right)}\right)(x-\breve{x})(y-\breve{y}) \\
-u_{4}(y-\breve{y})^{2}+(x-\breve{x})(z-\breve{z})-u_{5} u_{6}(z-\breve{z})^{2}
\end{gathered}
$$

Then by using the conditions $(9 a)-(9 c)$ we get.

$$
\frac{d V 1}{d t}<-\beta_{1}-\beta_{2} \text { Where, }
$$


$\beta_{1}=\left[\frac{1}{\sqrt{2}} \sqrt{1-\frac{u_{1} \breve{y}}{u_{2}\left(u_{2}+\breve{x}\right)}}(x-\breve{x})-\sqrt{u_{4}}(y-\breve{y})\right]^{2}$
$\beta_{2}=\left[\frac{1}{\sqrt{2}} \sqrt{1-\frac{u_{1} \breve{y}}{u_{2}\left(u_{2}+\breve{x}\right)}}(x-\breve{x})-\sqrt{u_{5} u_{6}}(z-\breve{z})\right]^{2}$.

Then we obtain that $\frac{d V_{1}}{d t}$ is negative definite and hence $V_{1}$ is a Lyapunov function. Thus $E_{8}$ is a globally asymptotically stable in the Int $R_{+}^{3}$ of the xyz-space and the proof is complete.

Theorem 5: Assume that, the equilibrium point $E_{9}=(\hat{x}, \hat{y}, 0, \hat{w})$ of system (2) is locally asymptotically stable in the Int $R_{+}^{3}$ and the following conditions hold.

$\frac{u_{1} \hat{y}}{u_{2}\left(u_{2}+\hat{x}\right)}<1$

$\left(\frac{e u_{1} u_{2}-u_{1} u_{2}-u_{1} \hat{x}}{\left(u_{2}-x\right)\left(u_{2}+\hat{x}\right)}\right)^{2}<4 u_{4}\left(1-\frac{u_{1} \hat{y}}{u_{2}\left(u_{2}+\hat{x}\right)}\right)$

Then the equilibrium point $E_{9}$ of the system (2) is globally asymptotically stable in the $I n t \cdot R_{+}^{3}$ of the xyw-space and the proof is complete. .

Proof: We will proof the theorem in the Int $\cdot R_{+}^{3}$. Clearly for any initial value in the Int $R_{+}^{3}$ of xyw-space, system (2) reduces to the following subsystem.

$$
\begin{aligned}
& \frac{d x}{d t}=x\left[(1-x)-\frac{u_{1} y}{u_{2}+x}\right]=x f_{1}(x, y, w) \\
& \frac{d y}{d t}=y\left[\frac{e u_{1} x}{u_{2}+x}-u_{3}-u_{4} y\right]=y f_{2}(x, y, w) \\
& \frac{d w}{d t}=w\left[u_{7}\left(1-u_{8} w\right)\right]=w f_{3}(x, y, w)
\end{aligned}
$$

Now, consider the following function

$$
V_{2}(x, y, z, w)=\left(x-\hat{x}-\hat{x} \ln \frac{x}{\hat{x}}\right)+\left(y-\hat{y}-\hat{y} \ln \frac{y}{\hat{y}}\right)+\left(w-\hat{w}-\hat{w} \ln \frac{w}{\hat{w}}\right)
$$

Clearly, $V_{2}: R_{+}^{3} \rightarrow R$ is a $C^{1}$ positive definite function? Now by differentiating $V_{2}$ with respect to time $\mathrm{t}$ and doing some algebraic manipulation, gives that:

$$
\begin{gathered}
\frac{d V_{2}}{d t} \leq-\left(1-\frac{u_{1} \hat{y}}{u_{2}\left(u_{2}+\hat{x}\right)}\right)(x-\hat{x})^{2}+\left(\frac{e u_{1} u_{2}-u_{1} \hat{x}-u_{1} u_{2}}{\left(u_{2}-x\right)\left(u_{2}+\hat{x}\right)}\right)(x-\hat{x})(y-\hat{y}) \\
-u_{4}(y-\hat{y})^{2}-u_{7} u_{8}(w-\hat{w})^{2}
\end{gathered}
$$

By using the condition (10a) and (10b) we get

$$
\frac{d V_{2}}{d t}<-\delta_{1}-\delta_{2}
$$

Where, $\delta_{1}=\left[\sqrt{1-\frac{u_{1} \hat{y}}{u_{2}\left(u_{2}+\hat{x}\right)}}(x-\hat{x})-\sqrt{u_{4}}(y-\hat{y})\right]^{2} ; \delta_{2}=u_{7} u_{8}(w-\hat{w})^{2}$.

Then $\frac{d V_{2}}{d t}$ is negative definite and hence $V_{2}$ is a Lyapunov function. Thus $E_{9}$ is a globally asymptotically stable in the Int $R_{+}^{3}$ of xyw-space and the proof is complete. 
Theorem 6: Assume that, the equilibrium point $E_{10}=\left(x^{\bullet}, 0, z^{\bullet}, w^{\bullet}\right)$ of system (2) is locally asymptotically stable in the Int $R_{+}^{3}$ then it is globally asymptotically stable.

Proof: We will proof the theorem in the . For any initial value clearly in the Int $R_{+}^{3}$ of xzW-space, system (2) reduces to the following subsystem.

$$
\begin{aligned}
& \frac{d x}{d t}=x[(1-x)+z]=x f_{1}(x, z, w) \\
& \frac{d z}{d t}=z\left[u_{5}\left(1-u_{6} z\right)-w\right]=z f_{3}(x, z, w) \\
& \frac{d w}{d t}=w\left[u_{7}\left(1-u_{8} w\right)-u_{9} z\right]=w f_{3}(x, z, w)
\end{aligned}
$$

Consider the following function

$V_{3}(x, y, z, w)=\left(x-x^{\bullet}-x \cdot \ln \frac{x}{x^{\bullet}}\right)+\left(z-z^{\bullet}-z^{\cdot} \ln \frac{z}{z^{\bullet}}\right)+\left(w-w^{\bullet}-w^{\bullet} \ln \frac{w}{w^{\bullet}}\right)$

Clearly, $V_{3}: R_{+}^{3} \rightarrow R$ is a $C^{1}$ positive definite function? Now by differentiating $V_{3}$ with respect to time $t$ and doing some algebraic manipulation, gives that:

$$
\begin{aligned}
\frac{d V_{3}}{d t} & \leq-\left(x-x^{\bullet}\right)^{2}+\left(x-x^{\bullet}\right)\left(z-z^{\bullet}\right)-u_{5} u_{6}\left(z-z^{\bullet}\right)^{2}-\left(z-z^{\bullet}\right)\left(w-w^{\bullet}\right)-u_{7} u_{8}\left(w-w^{\bullet}\right)^{2} \\
& -u_{9}\left(w-w^{\bullet}\right)\left(z-z^{\bullet}\right)
\end{aligned}
$$

Thus,

$$
\frac{d V_{3}}{d t} \leq-\left[(x-x \cdot)-\sqrt{\frac{u_{5} u_{6}}{2}}\left(z-z^{\cdot}\right)\right]^{2}-\left[\sqrt{\frac{u_{5} u_{6}}{2}}(z-z \cdot)-\sqrt{u_{7} u_{8}}(w-w \cdot)\right]^{2}
$$

Then $\frac{d V_{3}}{d t}$ is negative definite and hence $V_{3}$ is a Lyapunov function. Thus $E_{10}$ is a globally asymptotically stable in the Int.$R_{+}^{3}$ of XzW-space and the proof is complete.

Theorem 7: Assume that there are no periodic dynamics of system (2)in the boundary of the solution. Further, if the following conditions are held:

$$
\begin{aligned}
& e u_{1} \breve{x}>\left(u_{2}+\breve{x}\right)\left(u_{3}+u_{4} \breve{y}\right) \\
& \hat{x}+\frac{u_{1} \hat{y}}{u_{2}+\hat{x}}<1 \\
& u_{3}+u_{4} \hat{y}<\frac{e u_{1} \hat{x}}{u_{2}+\hat{x}} \\
& u_{5} u_{9}>1 \\
& u_{8}<u_{9} \\
& u_{5} u_{6} u_{7} u_{8}>u_{9} \\
& u_{6} u_{7}>u_{9} \\
& u_{8} w^{\cdot}<1 \\
& u_{9} z^{\cdot}<u_{7}\left(1-u_{8} w^{\cdot}\right)
\end{aligned}
$$


And

$M_{1}>M_{2}$

where,

$M_{1}=P_{2}\left[\frac{e u_{1} x^{\bullet}-u_{3}\left(u_{2}+x^{\bullet}\right)}{u_{2}+x^{\bullet}}\right]+P_{4}\left[u_{7}-u_{7} u_{8} w^{\bullet}-u_{9} z^{\bullet}\right]$ and

$M_{2}=P_{3}\left[\frac{u_{9}\left(u_{5}+1\right)}{u_{5} u_{6} u_{7} u_{8}-u_{9}}\right]$,

Then system (2) is uniformly persist.

Proof: Consider the following average Lyapunov function $\delta(x, y, z, w)=x^{P_{1}} y^{P_{2}} z^{P_{3}} w^{P_{4}}$, where each $p_{i}, i=1,2,3,4$ is assumed to be positive, obviously $\delta(x, y, z, w)$ is continuously differentiable positive function defined in, since.

$$
\begin{aligned}
& \Psi(x, y, z, w)=\frac{\delta^{\prime}(x, y, z, w)}{\delta(x, y, z, w)} \\
& =P_{1}\left[(1-x)-\frac{u_{1} y}{u_{2}+x}+z\right]+P_{2}\left[\frac{e u_{1} x}{u_{2}+x}-u_{3-u_{4} y}\right] \\
& +P_{3}\left[u_{5}\left(1-u_{6} z\right)-w\right]+P_{4}\left[u_{7}\left(1-u_{8} w\right)-u_{9} z\right]
\end{aligned}
$$

And, also it is assumed that there are no periodic attractors in the boundary plane, boundary space, and the equilibrium points $E_{0}-E_{5}$ are unstable points hence these equilibrium poins do not belong to the possible omega limit set of system (2), then the only possible omega limits set of system (2) are the equilibrium points. The proof is follows so nd the sysfollowed ormly persists if we can propersistedat each of these equilibium points.

1) For $E_{6}=(\bar{x}, 0, \bar{z}, 0)=\left(\frac{u_{6}+1}{u_{6}}, 0, \frac{1}{u_{6}}, 0\right)$ we have

$$
\Psi\left(E_{6}\right)=P_{2}\left[\frac{e u_{1}\left(1+u_{6}\right)-u_{3}\left(u_{2} u_{\left.6+u_{6}+1\right)}\right.}{u_{2} u_{6}+u_{6}+1}\right]+P_{4}\left[u_{7}\left(1-u_{8} w\right)-u_{9} z\right]
$$

Violate condition (2.3a) imply that $\Psi\left(E_{6}\right)>0$ for any $P_{2}>0$ and $P_{4}>0$.

2) For $E_{7}=(\overline{\bar{x}}, 0,0, \overline{\bar{w}})=\left(1,0,0, \frac{1}{u_{8}}\right)$ we have

$$
\Psi\left(E_{7}\right)=P_{2}\left[\frac{e u_{1}-u_{3}\left(u_{2+1)}\right.}{u_{2}+1}\right]-P_{3} \frac{1}{u_{8}} .
$$

Violate condition (4a) imply that $\Psi\left(E_{7}\right)>0$ for any $P_{2}>0$ and $P_{3}>0$.

3) For $E_{8}=(\breve{x}, \breve{y}, \breve{z}, 0)$ we have

$$
\Psi\left(E_{8}\right)=P_{2}\left[\frac{e u_{1} \breve{x}-u_{3}\left(u_{2}+\widetilde{x}\right)-u_{4} \breve{y}\left(u_{2}+\breve{x}\right)}{u_{2}+\widetilde{x}}\right] .
$$

So, $\Psi(E 8)>0$ for any $P_{2}>0$, provided conditions (11a) hold.

4) For $E_{9}=(\hat{x}, \hat{y}, 0, \hat{w})$ we have

$$
\Psi\left(E_{9}\right)=P_{1}\left[1-\left(\hat{x}+\frac{u_{1} \hat{y}}{u_{2}+\hat{x}}\right)\right]+P_{2}\left[\frac{e u_{1} \hat{x}}{u_{2}+\hat{x}}-\left(u_{3}+u_{4} \hat{y}\right)\right]+P_{3}\left[\frac{u_{5} u_{9-1}}{u_{9}}\right]+P_{4}\left[\frac{u_{7}\left(u_{9}-u_{8}\right)}{u_{9}}\right] .
$$

So, $\Psi\left(E_{9}\right)>0$ for any positive numbers $P_{1, P_{2}, P_{3}}$ and $P_{4}$ provided conditions $(11 \mathrm{~b})-(11 \mathrm{e})$ are hold with violate conditions (6a) and (6b). 
5) Finally for $E_{10}=\left(x^{\bullet}, 0, z^{\bullet}, w^{\bullet}\right)$ we have

$$
\Psi\left(E_{10}\right)=P_{2}\left[\frac{e u_{1} x^{\bullet} u_{3}\left(u_{2}+x^{\bullet}\right)}{u_{2}+x^{\bullet}}\right]+P_{3}\left[\frac{-u_{9}\left(u_{5}+1\right)}{u_{5} u_{6} u_{7} u_{8}-u_{9}}\right]+P_{4}\left[u_{7}\left(1-u_{8} w^{\bullet}\right)-u_{9} z^{\bullet}\right] .
$$

So, $\Psi\left(E_{10}\right)>0$ for any positive numbers $P_{2, P_{3}}$ and $P_{4}$ provided conditions (13f)-(13i) are holds with violate conditions (9b).

Hence system (2) is uniformly persists.

\section{Numerical simulation}

In this section, the dynamical behavior of system (2) is studied numerically for different sets of parameters and different sets of initial points. The objectives of this study are: first investigate the effect of varying the value of each parameter on the dynamical behavior of system (2) and second confirm our obtained analytical results. It is observed that, for the following set of hypothetical parameters that satisfies stability conditions of the positive equilibrium point, system (2) has a globally asymptotically stable positive equilibrium point as shown in Fig. (2).

Note that, from now onward the blue, green, red and sky blue colors are used to describing the trajectories of the prey $x$, the predator $y$, the host $z$ and the Host competitor $w$ respectively.

$$
\begin{gathered}
u_{1}=0.6, u_{2}=0.25, u_{3}=0.1, u_{4}=0.05, u_{5}=2, u_{6}=0.5 \\
u_{7}=2, u_{8}=0.75, u_{9}=0.8, e=0.5
\end{gathered}
$$

(a)
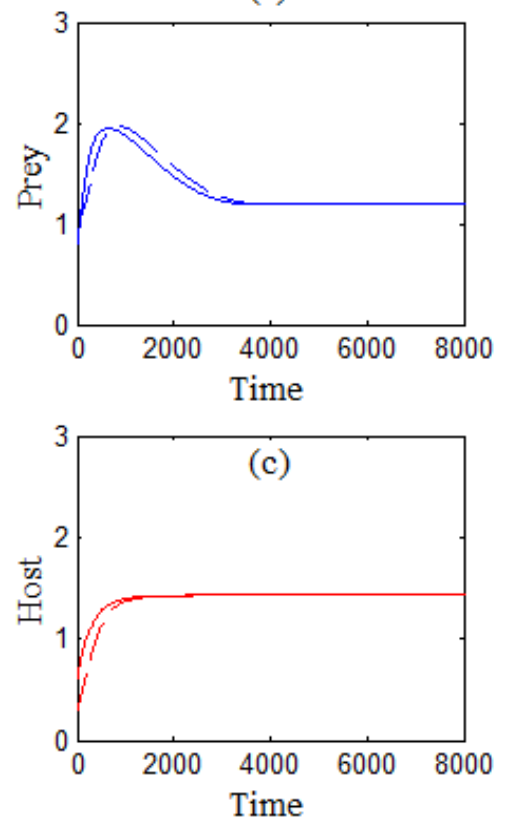

(b)

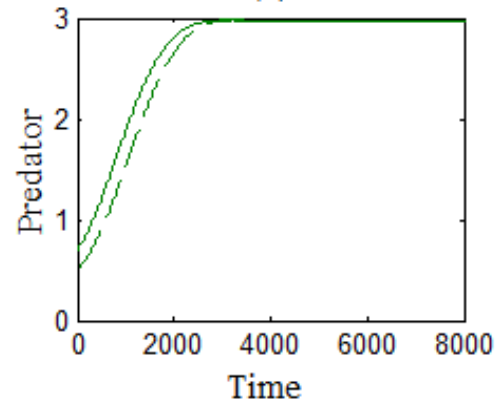

(d)

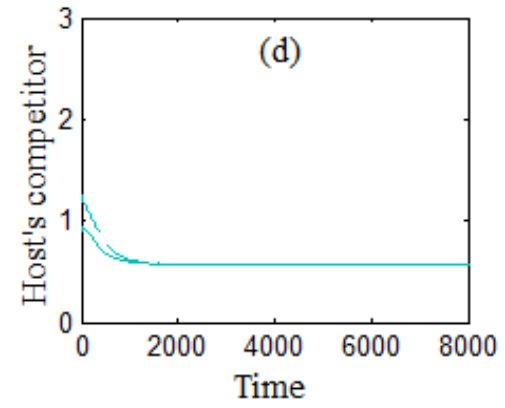

Fig. 1: Time Series of the Solution of System (2.2) That Started From Two Different Initial Points $(0.8,0.7,0.6,0.9)$ and $(1.0,0.5,0.3,1.25)$ for the Data Given by Eq. (14). (A) Trajectories of $x$ as A Function of Time, (B) Trajectories of $y$ as A Function of Time, (C) Trajectories of $z$ as A Function of Time,(D) Trajectories of $w$ as A Function of Time.

Clearly, Fig. (1) Shows that system (2) has a globally asymptotically stable as the solution of system (2) approaches asymptotically to the positive equilibrium point $E_{11}=(1.2,2.96,1.42,0.57)$ starting from two different initial points, and this is confirming our obtained analytical results. 


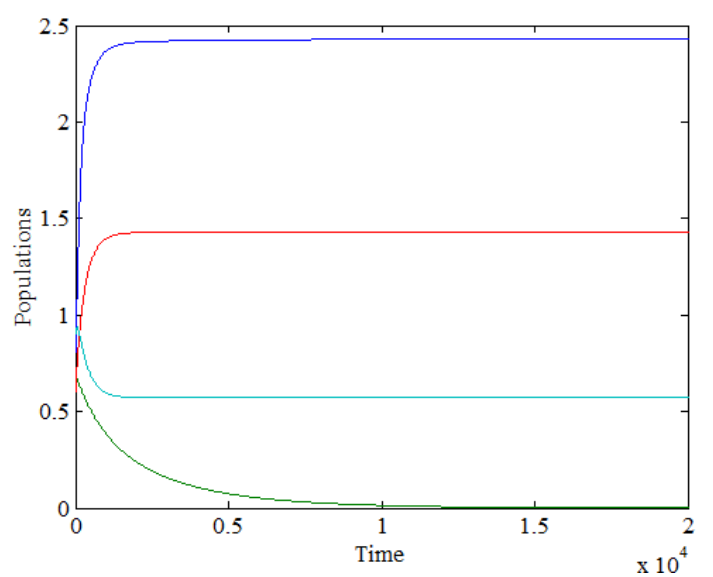

Fig. 2: Time Series of the Solution of System (2.2) for the Data Given by Eq. (14) with $u_{1}=0.15$, Which Approaches to $(2.42,0.0,1.42,0.57)$ in $x z w$ - Space.

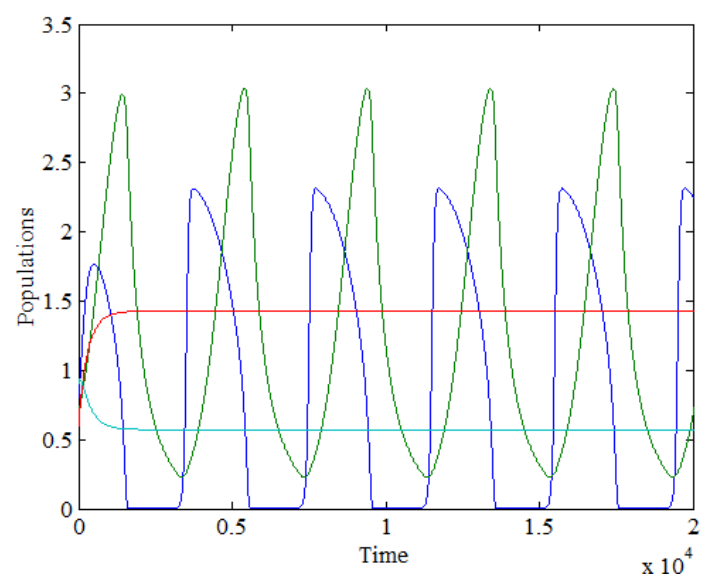

Fig. 3: Time Series of the Solution of System (2.2) for the Data Given by Eq. (14) with $u_{1}=0.7$, which Approaches to Periodic Dynamics in Int. $R_{+}^{4}$.

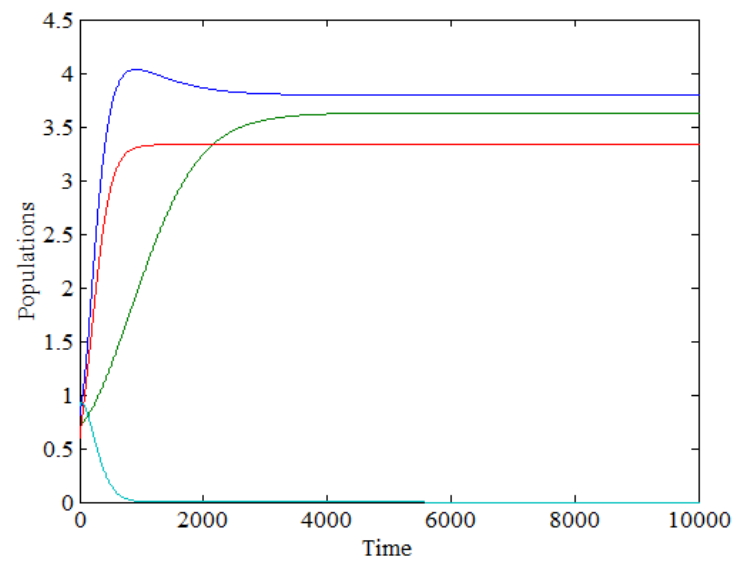

Fig. 4: Time Series of the Solution of System (2) for the Data Given by Eq. (14) with $u_{6}=0.3$, which Approaches Asymptotically to $(3.79,3.62,3.33,0)$ in the Interior of Positive Octant of $x y z-$ Space.

Fig. (5): Time series of the solution of system (2) for the data given by Eq. (14) with $e=0.1$, which approaches asymptotically to $(2.42,0,1.42,0.57)$ in the interior of a positive octant of $x z w$ - space.

According to the above, the effect of the other parameters on the dynamics of system (2) is also studied in case of varying the parameters and obtained results are summarized in the following tables. 
Table 1: Numerical Behaviors and Persistence of System (3) as Varying in Some Parameters Keeping the Rest of Parameters Fixed as in Eq. (14).

\begin{tabular}{|c|c|c|}
\hline Parameters varied in system(2) & Numerical behavior of system (2) & Persistence of system (2) \\
\hline $0.1 \leq u_{1}<0.23$ & $\begin{array}{l}\text { Approaches to stable point in Int. } R_{+}^{3} \\
\text { Approaches to the positive stable point } E_{11}\end{array}$ & $\begin{array}{l}\text { Not Persist } \\
\text { persist }\end{array}$ \\
\hline$u_{2}<0.24$ & $\begin{array}{l}\text { Approaches to periodic dynamics in Int. } R_{+}^{4} \\
\text { Approaches to the positive stable point } E_{11}\end{array}$ & $\begin{array}{l}\text { persist } \\
\text { persist }\end{array}$ \\
\hline$u_{3} \leq 0.09$ & Approaches to periodic dynamics in Int. $R_{+}^{4}$ & Persist \\
\hline $0.09<u_{3}<0.27$ & Approaches to the positive stable point $E_{10}$ & Not persist \\
\hline$u_{4} \leq 0.04$ & Approaches to periodic dynamics in Int. $R_{+}^{4}$ & Persist \\
\hline $0.04<u_{4}<1$ & Approaches to the stable point $E_{10}$ & Persist \\
\hline $1.33<u_{5}<1.86$ & $\begin{array}{l}\text { Approaches to periodic dynamics in Int. } R_{+}^{4} \\
\text { Approaches to the positive stable point } E_{11}\end{array}$ & $\begin{array}{l}\text { Persist } \\
\text { persist }\end{array}$ \\
\hline$u_{6} \leq 0.4$ & Approaches to stable point in $E_{8}$ & Not Persist \\
\hline$u_{6}>0.4$ & Approaches to the positive stable point $E_{11}$ & Persist \\
\hline$u_{7} \leq 1.6$ & Approaches to the stable point $E_{8}$ & Not Persist \\
\hline $1.6<u_{7} \leq 2.1$ & Approaches to the positive stable point $E_{11}$ & Persist \\
\hline$u_{7}>2.1$ & Approaches to periodic dynamics in Int. $R_{+}^{4}$ & Persist \\
\hline$u_{8} \leq 0.5$ & Approaches to periodic dynamics in Int. $R_{+}^{3}$ & Not Persist \\
\hline$u_{8}>0.5$ & Approaches to the positive stable point $E_{11}$ & Persist \\
\hline$u_{9}<0.75$ & Approaches to periodic dynamics in Int. $R_{+}^{4}$ & Persist \\
\hline $0.75 \leq u_{9}<0.99$ & Approaches to the positive stable point $E_{11}$ & Persist \\
\hline$u_{9} \geq 0.99$ & Approaches to the stable point $E_{8}$ & Not Persist \\
\hline$e \leq 0.17$ & Approaches to the stable point $E_{10}$ & Not Persist \\
\hline $0.17 \leq e<0.51$ & Approaches to the positive stable point $E_{11}$ & Persist \\
\hline$e>0.51$ & Approaches to periodic dynamics in Int. $R_{+}^{4}$ & Persist \\
\hline
\end{tabular}

\section{References}

[1] Manju A.and RachanaP.2012.Persistence and optimal harvesting of prey-predator model with Holling Type III functional responce, International Journal of Engineering,Science and Technology,Vol.4,No.3,pp.78-96.

[2] Debasis M.2012.Persistence in a generalized prey-predator model with prey reserve,International Journal of Nonlinear Science,Vol.14,No.2,pp.160-165.

[3] T.K.Kar and Ashim B.2010.Persistence and stability of a two prey one predator system, International Journal of Engineering,Science and Technology,Vol.2,No.2,pp.174-190. http://dx.doi.org/10.4314/ijest.v2i2.59164.

[4] Chen, F. and You, M. 2008. Permanence, extinction and periodic solution of the predator-prey system with Beddington-DeAngelis functional respon-se and stage structure for prey. Nonlinear Analysis: Real World Applications, 9:207 - 221. http://dx.doi.org/10.1016/j.nonrwa.2006.09.009.

[5] Freedman H.I. and Ruan S., 1995.Uniform persistence in functional differential equations, J.Differential Equations 115,173-192. http://dx.doi.org/10.1006/jdeq.1995.1011.

[6] Chen F. 2006. Permanence of periodic Holling type predator-prey system with stage structure for prey, Applied Mathematics and Computation, 182(2):1849-1860. http://dx.doi.org/10.1016/j.amc.2006.06.024.

[7] Cui J. and Song, X. 2004. Permanence of predator-prey system with stage structure, Discrete and Continuous Dynamical System. Series B, 4(3):547-554. http://dx.doi.org/10.3934/dcdsb.2004.4.547.

[8] B.Dubey and R.K.Upadhyay 2004.Persistence and extinction of one-prey and two predators system, Nonlinear Analysis:Modelling and Control,Vol.9,No.4,307-329.

[9] Freedman H.I. and Waltman P., 1984.Persistence in modeles of three are interacting predator-prey populations, Math.Biosci, 68,213-231. http://dx.doi.org/10.1016/0025-5564(84)90032-4.

[10] Rami Raad \& Raid K.Naji. 2014. The dynamics of the multi-interactions ecological systems,Lambert Academic Publishing.

[11] Freedman H.I. and Ruan S., 1995.Uniform persistence in functional differential equations, J.Differential Equations 115,173-192. http://dx.doi.org/10.1006/jdeq.1995.1011.

[12] Gard T.C.and Hallam T.G., 1979. Persistence in food webs-I.Lotka Volterra food chains, Bull. Math.Biol.,41,877-891 http://dx.doi.org/10.1016/S0092-8240(79)80024-5. 